\title{
Enhancement of Recombinase-Aided Amplification (Raa) Assay by Betaine and Pullulan
}

\section{Jinrong Wang}

Hebei Medical University

\section{Guowei Song}

Shijiazhuang People's Hospital

\section{Yue Ming}

Qiqihar Medical University

\section{Jing Pan}

Shijiazhuang People's Hospital

\section{Ruiqing Zhang}

Chinese Center for Disease Control and Prevention

\section{Guohao Fan}

Chinese Center for Disease Control and Prevention

\section{Yuan Gao}

Chinese Center for Disease Control and Prevention

\section{Xinxin Shen}

Chinese Center for Disease Control and Prevention

\section{Xuejun Ma ( $\nabla$ maxj@ivdc.chinacdc.cn )}

Chinese Center for Disease Control and Prevention

\section{Lixin Li}

Shijiazhuang People's Hospital

\section{Research Article}

Keywords: Nucleic acid amplification enhancer, Betaine, Pullulan, RAA, Long-fragment

Posted Date: May 7th, 2021

DOl: https://doi.org/10.21203/rs.3.rs-463676/v1

License: () (i) This work is licensed under a Creative Commons Attribution 4.0 International License.

Read Full License 


\section{Abstract}

In this research, nucleic acid amplification enhancers suitable for recombinase-aided amplification (RAA) assay were studied for the first time, and amplification of a long-fragment (509 bp) by the RAA assay was initially explored. Using recombinant plasmids and clinical samples, RAA fluorescence and basic methods were used to evaluate the efficacy. The fluorescence method was evaluated by threshold time (TT) and fluorescence value, and the basic method was interpreted by $2 \%$ agarose gel electrophoresis. Taking a previously established RAA assay for HPV18 as an example, we demonstrated that the addition of $0.2,0.4$, and $0.6 \mathrm{M}$ betaine and $10 \%$ pullulan could enhance RAA. The new RAA assays with betaine and pullulan were called B-RAA and P-RAA, respectively. In the B-RAA and P-RAA fluorescence methods, TT values could be shortened by $1.72-2.32$ and 2.60 minutes, respectively, and fluorescence values could be enhanced by 8847.25-9094.37 and $5250 \mathrm{mv}$, respectively. In the basic method, sensitivity could be increased by 10fold. We successfully amplified a long-fragment of $509 \mathrm{bp}$ in a P-RAA assay with a sensitivity of $10^{2}$ copies/ $\mu \mathrm{L}$ (compared with $10^{3}$ copies/ $\mu \mathrm{L}$ in the RAA assay). We thus conclude that betaine and pullulan are effective additives to enhance RAA assays.

\section{Introduction}

Recombinase-aided amplification (RAA) assay is a new technique for nucleic acid amplification under isothermal conditions that employs recombinase, single-stranded DNA binding (SSB), and DNA polymerase [1]. The procedure is usually conducted at $37^{\circ} \mathrm{C}-42^{\circ} \mathrm{C}$. RAA combines recombinase obtained from bacteria or fungi with specific primers to form an enzyme-primer complex. When this complex locates its complementary sequence on the template DNA, the template DNA is unlinked with the help of SSB, and a new complementary chain is formed under the action of DNA polymerase. The RAA method can achieve amplification within 20 min and, thus, presents the advantages of short reaction times and simple operation procedures over traditional PCR assays, as illustrated in previous reports on the detection of SARS-CoV-2 (e.g., Xue G et al. [2], Wu T et al.[3], and Wang $J$ et al.[4]) and other pathogens [5-9].

Although RAA is a promising isothermal nucleic acid detection method, the primer and probe designs for this technique are more complex than those for PCR primers and probes because RAA requires primers and probes with greater lengths. The design of RAA primers and probes is closely related to the detection sensitivity and specificity of the assay. Utilization of degenerate bases or mismatches in the probe or primer sequence could reduce the sensitivity and increase the non-specificity of amplification. Another limitation of the RAA assay is the presence of relatively short amplicons (100-200 bp, referred to as shortsegment amplification in this paper). To address these issues, we attempted to explore some chemical additives to improve the sensitivity and specificity and overcome the amplicon size limitation of RAA assays.

We previously reported a conventional RAA fluorescence assay for HPV 18 with a sensitivity of 10 copies/ $\mu \mathrm{L}$ [10]. Taking this assay as an example, in the present study, we explored two chemical additives, namely, betaine and pullulan polysaccharides, as potential enhancers for RAA. The new RAA methods added with 
these two enhancers are called B-RAA and P-RAA, respectively. We also examined the RAA of a $\sim 500 \mathrm{bp}$ template (referred to as long-fragment amplification in this paper) with the addition of these two enhancers.

\section{Materials And Methods}

\subsection{Materials and reagents}

The RAA probes and primers used for HPV18 detection are described in detail in our previous report [10] and listed in Table 1. The plasmid was cloned by TsingKe Biotech Corp. (Beijing, China). Two RAA nucleic acid amplification kits, i.e., the fluorescence and basic methods, were purchased from Qitian Biological Co., Ltd. (Jiangsu, China).

\subsection{Original RAA reaction}

\subsubsection{Original RAA fluorescence method}

In the RAA fluorescence method, the RAA nucleic acid amplification kit (fluorescence method) was used to configure a reaction system of $50 \mu \mathrm{L}$. The system consisted of $25 \mu \mathrm{L}$ of reaction buffer, $2.1 \mu \mathrm{L}$ of each forward and reverse primer $(10 \mu \mathrm{M}), 0.6 \mu \mathrm{L}$ of the HPV18 probe $(10 \mu \mathrm{M}), 1 \mu \mathrm{L}$ of 10 copies $/ \mu \mathrm{L}$ plasmid template or DNase-free water (negative control), and $2.5 \mu \mathrm{L}$ of $280 \mathrm{mM}$ magnesium acetate. The reaction tube was incubated in an isothermal oscillatory homogenizer (RAA-B6100) at $39^{\circ} \mathrm{C}$ for 4 min, briefly swayed and centrifuged, and then transferred to a constant-temperature nucleic acid amplification detector (RAA-F1620) for $30 \mathrm{~min}$ at $39.0^{\circ} \mathrm{C}$. The results were observed in real-time.

\subsubsection{Original RAA basic method}

In the original RAA basic method, the RAA nucleic acid amplification kit (basic method) was used to configure a reaction system of $50 \mu \mathrm{L}$. The system, operating, and reaction conditions are identical to those described above for the fluorescence method, but no probe was added to the system. Agarose gel (Beijing Rocco Xinye Biotechnology Co., Ltd.) was stained with SYBR Green I nucleic acid dye (10000x, electrophoresis grade; Solarbio, Beijing, China). Next, $2 \mu \mathrm{L}$ of $5 \times$ Green Gotaq Flexi Buffer (Promega, USA) was mixed with $8 \mu \mathrm{L}$ of the product purified by DNA extraction (phenol:chloroform:isoamyl alcohol = 25:24:1; pH > 7.8) or DL2000TM DNA Marker (TaKaRa, Japan) for $2 \%$ agarose gel electrophoresis and visualized with the Gel DocTMXR + Imaging System (Bio-Rad, USA).

\subsection{B-RPA reaction}

\subsubsection{B-RAA fluorescence method}

The reaction was identical to the original RAA fluorescence method, except that betaine (Aladdin, Shanghai, China) was added at various final concentrations of $0.2,0.4,0.6,0.8$, and $1.0 \mathrm{M}$. Eight replicates of each reaction were performed to evaluate the repeatability of the B-RAA method.

\subsubsection{B-RAA basic method}


The reaction was identical to the original RAA basic method. The addition of betaine was conducted as described for the B-RAA fluorescence method.

\subsection{P-RPA reaction}

\subsubsection{P-RAA fluorescence method}

The reaction was identical to that of the original RAA fluorescence method, except that different concentrations of pullulan (Sigma-Aldrich, USA) were added. The pullulan additive was prepared as follows. Exactly $0.1 \mathrm{~g}$ of pullulan was added to a centrifuge tube containing $1 \mathrm{~mL}$ of deionized water and placed in an ultrasonic microwave cleaner several times to accelerate dissolution and prepare a 10\% $(\mathrm{w} / \mathrm{v})$ stock solution. The prepared pullulan stock solution was serially diluted 10 times to obtain solutions of 1$10 \%$, and $1 \mu \mathrm{L}$ was added to different unit tubes [11].

\subsubsection{P-RAA basic method}

The reaction was identical to the original RAA basic method. Pullulan was added as described for the PRAA fluorescence method.

\subsection{Long-fragment (500 bp) amplification by the original RAA, B-RAA, and P-RAA assays}

Using the probe and reverse primers designed in our previous work [10], we redesigned a forward primer (Table 1) to increase the length of the amplification product to $509 \mathrm{bp}$. The amplification method was identical to that in B-RAA and P-RAA. In the RAA long-fragment (509 bp) fluorescence and basic methods, we used the original RAA, B-RAA, and P-RAA assays to amplify $10^{2}-10^{4}$ copies/ $\mu \mathrm{L}$ HPV 18 plasmids and repeated the experiments eight times. We added $0.4 \mathrm{M}$ betaine to B-RAA and $10 \%$ pullulan to P-RAA.

Table 1

Primers and probes used for RAA. ${ }^{2}$ Probe: FAM, 6-carboxyfuorescein; THF, tetrahydrofuran; BHQ, black hole quencher; C3Spacer, 3' phosphate blocker.

\begin{tabular}{|c|c|c|c|}
\hline $\begin{array}{l}\text { Primers } \\
\text { and Probes }\end{array}$ & Sequences $\left(5^{\prime}-3^{\prime}\right)$ & $\begin{array}{l}\text { Amplicon } \\
\text { Size }\end{array}$ & Source \\
\hline $\begin{array}{l}\text { HPV18- } \\
\text { Forward }\end{array}$ & TCTACCATATCCGACCCTGTGTCTGTTGCAT & $509 \mathrm{bp}$ & $\begin{array}{l}\text { This } \\
\text { paper }\end{array}$ \\
\hline $\begin{array}{l}\text { HPV18- } \\
\text { Reverse }\end{array}$ & ACTCCAACGACGCAGAGAAACACAAGTATAA & & [10] \\
\hline $\begin{array}{l}\text { HPV18- } \\
\text { Probe }^{\text {a }}\end{array}$ & $\begin{array}{l}\text { ATTTCATCGTTTTCTTCCTCTGAGTCGCT[FAM-dT] } \\
\text { [dSpacer]A[BHQ-dT]TGCTCGTGACATA[C3-spacer] }\end{array}$ & & [10] \\
\hline
\end{tabular}


We selected the nucleic acids of $21 \mathrm{HPV} 18$ positive samples (cervical exfoliated cells) preserved by the NHC Key Laboratory of Medical Virology and Viral Diseases, National Institute for Viral Disease Control and Prevention, Chinese Center for Disease Control and Prevention. These samples were tested positive for HPV18 by Cobas 4800 HPV from August 2019 to December 2019. In this work, we amplified short and long fragments by using the original RAA, B-RAA, and P-RAA fluorescence methods. The amounts of nucleic acid added to the sample during short- and long-fragment amplification were 2 and $5 \mu \mathrm{L}$, respectively. In addition, 0.6 and $0.4 \mathrm{M}$ betaine were respectively used for short- and long-fragment amplification via B-RAA. The concentration of pullulan used in P-RAA was $10 \%$.

\section{Results}

\subsection{B-RAA reaction}

\subsubsection{B-RAA fluorescence method}

We repeated the B-RAA fluorescence method eight times. The average threshold time (TT, minute) was shortened, and the average fluorescence value was enhanced by different betaine concentrations when compared with those obtained from the original RAA fluorescence method without the addition of betaine. The addition of $0.2,0.4$, and $0.6 \mathrm{M}$ betaine could enhance the RAA reaction, shorten the TT value by $1.72-$ $2.32 \mathrm{~min}$, enhance the fluorescence value by $8847.25-9094.37 \mathrm{mv}$ (Table 2), and increase the amount of RAA amplification products. However, the addition of 0.8 or $1.0 \mathrm{M}$ betaine inhibited the RAA assay, as shown in Fig. 1A. 
Table 2

Results of the B-RAA fluorescence method with different betaine concentrations, the P-RAA fluorescence method with different pullulan concentrations, and the average TT and fluorescence values of eight repeat experiments.

\begin{tabular}{|llll|}
\hline \multicolumn{1}{|l}{ Final concentration } & Means of TT values $(\mathbf{m i n})$ & Means of fluorescence $(\mathrm{mv})$ \\
\hline Betaine (M) & 0 (original RAA) & 7.45 & 12998.38 \\
\cline { 2 - 4 } & 0.2 & 5.73 & 22092.75 \\
\cline { 2 - 3 } & 0.4 & 5.29 & 21985.63 \\
\hline 0.6 & 5.13 & 21845.63 \\
\hline 0.8 & 6.46 & 15492.25 \\
\hline 1.0 & 6.69 & 15906.13 \\
\hline Pullulan (\%) & 0 (original RAA) & 8.02 & 12774.88 \\
\hline 2 & 7.46 & 13475.25 \\
\hline 4 & 8.19 & 13411.50 \\
\hline 6 & 7.29 & 12532.63 \\
\hline 10 & 8.08 & 13515.00 \\
\hline
\end{tabular}

\subsubsection{B-RAA basic method}

The amplification products of the RAA basic method were electrophoretic on an agarose gel. In the absence of betaine, no amplification bands were generated from 10 copies/ $\mu \mathrm{L}$ HPV 18 plasmids. However, when the final concentration of betaine was $0.2,0.4$, or $0.6 \mathrm{M}$, the amplification bands and a few nonspecific bands were observed (Fig. 1B).

\subsection{P-RAA reaction.}

\subsubsection{P-RAA fluorescence method}

We repeated the P-RAA fluorescence method eight times. Compared with the original RAA fluorescence method without pullulan addition, RAA fluorescence with $10 \%$ pullulan could steadily enhance the RAA assays by shortening the TT and increasing the fluorescence values. P-RAA could shorten the TT value by $2.60 \mathrm{~min}$, enhance the fluorescence value by $5250 \mathrm{mv}$ (Fig. 1C, Table 2), and increase the amount of RAA amplification products.

\subsubsection{P-RAA basic method}

Products amplified by the RAA basic method were electrophoretic on an agarose gel. Without pullulan, no band was amplified in the presence of 10 copies/ $\mu \mathrm{L} \mathrm{HPV} 18$ plasmids. At pullulan concentrations of $2 \%$, 
$4 \%, 8 \%$, and $10 \%$, the target fragment was successfully amplified, and only a few non-specific bands were generated. The amplification effects improved when $8 \%$ and $10 \%$ pullulan were applied. Therefore, the addition of pullulan to the RAA basic method can also increase the sensitivity of the technique by 10 -fold and reduce non-specific amplification (Fig. 1D).

\subsection{Long-fragment ( $509 \mathrm{bp}$ ) amplification by original the RAA, B-RAA, and P-RAA assays}

As shown in Fig. 1E, the amplification sensitivity of the original RAA and B-RAA could reach $10^{3}$ copies $/ \mu \mathrm{L}$, while the sensitivity of P-RAA could reach $10^{2}$ copies/ $\mu \mathrm{L}$. Moreover, the fluorescence values of the amplification results under different concentrations of the plasmid were higher than those of the two other RAA methods, and the TT value was shortened in P-RAA (Table 3). The enhancement effect of B-RAA on long-fragment amplification was not obvious. 
Table 3

In the 509 bp long-fragment amplification, the original RAA, B-RAA, and P-RAA amplified the HPV18 plasmid with a concentration of $10^{2}-10^{4}$ copies $/ \mu \mathrm{L}$ for eight times.

\begin{tabular}{|c|c|c|c|c|c|c|c|}
\hline \multicolumn{2}{|c|}{$\begin{array}{l}\text { Plasmid concentration } \\
\text { Type }\end{array}$} & \multicolumn{2}{|c|}{$10^{2}$ copies $/ \mu \mathrm{L}$} & \multicolumn{2}{|c|}{$10^{3}$ copies $/ \mu \mathrm{L}$} & \multicolumn{2}{|c|}{$10^{4}$ copies $/ \mu \mathrm{L}$} \\
\hline & & TT & Fluorescence & TT & Fluorescence & TT & Fluorescence \\
\hline \multirow[t]{8}{*}{ Original RAA } & 1 & negative & 1724 & 16.00 & 3116 & 11.33 & 7720 \\
\hline & 2 & negative & 1904 & 13.00 & 4252 & 7.67 & 10554 \\
\hline & 3 & negative & 1687 & 19.33 & 2419 & 6.00 & 6205 \\
\hline & 4 & negative & 1167 & 12.00 & 4645 & 6.67 & 9777 \\
\hline & 5 & negative & 999 & negative & 1215 & 11.33 & 8022 \\
\hline & 6 & negative & 1507 & 13.00 & 1742 & 6.33 & 13515 \\
\hline & 7 & negative & 1541 & 12.67 & 5644 & 6.33 & 13547 \\
\hline & 8 & negative & 1248 & 17.67 & 7117 & 9.33 & 6887 \\
\hline \multirow[t]{8}{*}{ B-RAA } & 1 & negative & 1521 & 19.00 & 2835 & 10.33 & 8363 \\
\hline & 2 & negative & 1292 & 16.67 & 2424 & 8.67 & 7886 \\
\hline & 3 & negative & 1581 & 17.00 & 2861 & 6.67 & 5740 \\
\hline & 4 & negative & 1611 & 16.67 & 2900 & 7.33 & 8194 \\
\hline & 5 & negative & 999 & negative & 1391 & 13.67 & 3397 \\
\hline & 6 & negative & 1259 & 19.33 & 2201 & 8.67 & 7302 \\
\hline & 7 & negative & 1357 & negative & 1623 & 7.67 & 9806 \\
\hline & 8 & negative & 1454 & 16.33 & 4233 & 12.33 & 8629 \\
\hline \multirow[t]{8}{*}{ P-RAA } & 1 & 12.67 & 4550 & 8.00 & 9469 & 3.00 & 16292 \\
\hline & 2 & 22.00 & 2790 & 12.00 & 6310 & 8.00 & 10568 \\
\hline & 3 & 18.67 & 2157 & 15.00 & 3055 & 6.33 & 6666 \\
\hline & 4 & 21.33 & 2199 & 10.67 & 4425 & 4.67 & 13957 \\
\hline & 5 & 14.33 & 5949 & 11.33 & 7130 & 8.33 & 10272 \\
\hline & 6 & 23.67 & 1723 & 11.67 & 6026 & 6.33 & 13076 \\
\hline & 7 & 19.00 & 2857 & 10.33 & 9280 & 6.00 & 15098 \\
\hline & 8 & 18.67 & 3848 & 13.33 & 7928 & 6.33 & 15719 \\
\hline
\end{tabular}


The sensitivity of the basic RAA method was lower than that of the fluorescence method. Amplification of $10^{2}-10^{4}$ copies/ $\mu$ L HPV 18 plasmids was achieved, and the product was subjected to $2 \%$ agarose gel electrophoresis. B-RAA revealed no amplification bands. In the original RAA and P-RAA assays, the electrophoretic results of $10^{4}$ copies/ $\mu \mathrm{L}$ plasmids showed an amplification product of $500 \mathrm{bp}$; some nonspecific bands were also observed in P-RAA (Fig. 1F). The use of $10 \%$ pullulan achieved the best enhancement effect on long-fragment amplification.

\subsection{Sample detection by the original RAA, B-RAA, and P-RAA assays}

During short-segment amplification, all samples could be detected by the three methods, and the detection results of the three RAA methods were consistent with our previous results [10]. The fluorescence values in B-RAA and P-RAA are higher than that in the original RAA, and the TT values in these assays were less than or equal to that in the original RAA in $85.71 \%(18 / 21)$ and $76.19 \%(16 / 21)$ of the tested samples, respectively. The fluorescence enhancement and TT value shortening effects in B-RAA were greater than those in P-RAA (Table 4).

During long-fragment (509 bp) amplification, the detection rates of the original RAA, B-RAA, and P-RAA assays were $66.67 \%$ (14/21), 61.90 (13/21), and 71.43\% (15/21), respectively. Among the 13 samples that could be detected by all three methods, the fluorescence values in B-RAA and P-RAA were higher than those in the original RAA, and the TT values in these assays were less than or equal to that in the original RAA in $7.69 \%(1 / 13)$ and $84.62 \%(11 / 13)$ of the tested samples, respectively (Table 5).

The results of sample detection were consistent with those of plasmid detection. Betaine showed excellent enhancement effects for short-segment amplification, whereas pullulan demonstrated favorable effects for long-segment amplification. 
Table 4

Results of short-segment amplification of HPV 18 specimens by the original RAA, B-RAA, and P-RAA fluorescence methods.

\begin{tabular}{|c|c|c|c|c|c|c|c|}
\hline \multirow[t]{2}{*}{ Number } & \multirow{2}{*}{$\begin{array}{l}\mathrm{Ct} \\
\text { value }\end{array}$} & \multicolumn{2}{|l|}{ Original RAA } & \multicolumn{2}{|l|}{ B-RAA (0.6 M) } & \multicolumn{2}{|l|}{ P-RAA (10\%) } \\
\hline & & $\begin{array}{l}\text { Fluorescence } \\
\text { (mv) }\end{array}$ & $\begin{array}{l}\text { TT } \\
\text { (min) }\end{array}$ & $\begin{array}{l}\text { Fluorescence } \\
(\mathrm{mv})\end{array}$ & $\begin{array}{l}\text { TT } \\
\text { (min) }\end{array}$ & $\begin{array}{l}\text { Fluorescence } \\
\text { (mv) }\end{array}$ & $\begin{array}{l}\text { TT } \\
\text { (min) }\end{array}$ \\
\hline 1 & 20.09 & 15429 & 4.33 & 25832 & 2.67 & 23086 & 4.00 \\
\hline 2 & 20.16 & 11727 & 2.33 & 24689 & 3.00 & 12274 & 1.67 \\
\hline 3 & 21.41 & 28466 & 0.33 & 32415 & 0.00 & 28152 & 0.00 \\
\hline 4 & 22.28 & 28367 & 1.00 & 32869 & 1.00 & 29730 & 1.00 \\
\hline 5 & 26.71 & 13647 & 4.33 & 16702 & 4.00 & 19329 & 3.33 \\
\hline 6 & 26.81 & 12376 & 6.33 & 21712 & 3.33 & 20346 & 2.67 \\
\hline 7 & 27.94 & 19018 & 1.33 & 29455 & 1.33 & 24064 & 1.00 \\
\hline 8 & 28.04 & 4835 & 11.00 & 16864 & 3.67 & 8981 & 6.00 \\
\hline 9 & 28.56 & 11297 & 5.00 & 15997 & 4.00 & 14973 & 4.00 \\
\hline 10 & 29.88 & 8679 & 8.67 & 11734 & 6.00 & 18261 & 4.33 \\
\hline 11 & 30.34 & 13602 & 4.33 & 27161 & 3.00 & 16398 & 2.67 \\
\hline 12 & 31.19 & 20413 & 1.33 & 26196 & 1.67 & 18777 & 1.00 \\
\hline 13 & 32.34 & 7848 & 7.33 & 10442 & 6.67 & 13753 & 4.33 \\
\hline 14 & 32.36 & 32436 & 2.00 & 32563 & 2.00 & 30830 & 1.67 \\
\hline 15 & 33.08 & 13573 & 5.67 & 6015 & 8.67 & 14492 & 3.33 \\
\hline 16 & 34.18 & 10949 & 2.34 & 15649 & 4.66 & 9963 & 2.66 \\
\hline 17 & 34.88 & 9365 & 7.67 & 12563 & 5.00 & 25729 & 4.67 \\
\hline 18 & 35.60 & 4332 & 8.00 & 18863 & 2.00 & 16913 & 0.67 \\
\hline 19 & 35.87 & 15852 & 4.33 & 16150 & 4.21 & 15734 & 3.67 \\
\hline 20 & 37.68 & 7969 & 10.00 & 15213 & 5.67 & 10768 & 7.67 \\
\hline 21 & 37.84 & 12469 & 4.67 & 17475 & 3.67 & 15380 & 3.33 \\
\hline
\end{tabular}


Table 5

Results of long-segment amplification of HPV 18 specimens by the original RAA, B-RAA, and P-RAA fluorescence methods.

\begin{tabular}{|c|c|c|c|c|c|c|c|}
\hline \multirow[t]{2}{*}{ Number } & \multirow{2}{*}{$\begin{array}{l}\mathrm{Ct} \\
\text { value }\end{array}$} & \multicolumn{2}{|l|}{ Original RAA } & \multicolumn{2}{|l|}{ B-RAA (0.4 M) } & \multicolumn{2}{|l|}{ P-RAA (10\%) } \\
\hline & & $\begin{array}{l}\text { Fluorescence } \\
\text { (mv) }\end{array}$ & $\begin{array}{l}\text { TT } \\
\text { (min) }\end{array}$ & $\begin{array}{l}\text { Fluorescence } \\
\text { (mv) }\end{array}$ & $\begin{array}{l}\text { TT } \\
\text { (min) }\end{array}$ & $\begin{array}{l}\text { Fluorescence } \\
\text { (mv) }\end{array}$ & $\begin{array}{l}\text { TT } \\
\text { (min) }\end{array}$ \\
\hline 1 & 20.09 & 15845 & 5.33 & 13178 & 7.33 & 22302 & 4.00 \\
\hline 2 & 20.16 & 14946 & 0.67 & 20435 & 0.00 & 21506 & 0.00 \\
\hline 3 & 21.41 & 17603 & 2.33 & 15228 & 4.67 & 18983 & 1.00 \\
\hline 4 & 22.28 & 16215 & 1.00 & 16247 & 2.33 & 17365 & 0.33 \\
\hline 5 & 26.71 & 9200 & 7.00 & 3726 & 11.67 & 8514 & 9.00 \\
\hline 6 & 26.81 & 11160 & 7.67 & 10625 & 7.67 & 11293 & 7.67 \\
\hline 7 & 27.94 & 14061 & 6.33 & 6773 & 7.67 & 15401 & 5.33 \\
\hline 8 & 28.04 & 9646 & 8.33 & 6191 & 9.33 & 9913 & 8.00 \\
\hline 9 & 28.56 & 4049 & 10.67 & 3532 & 11.33 & 4497 & 10.00 \\
\hline 10 & 29.88 & 7413 & 7.67 & 4587 & 10.00 & 8244 & 5.67 \\
\hline 11 & 30.34 & 1522 & negative & 1214 & negative & 2150 & negative \\
\hline 12 & 31.19 & 8136 & 7.00 & 5091 & 9.33 & 9775 & 7.33 \\
\hline 13 & 32.34 & 2319 & 17.67 & 2088 & 18.00 & 3972 & 12.00 \\
\hline 14 & 32.36 & 2369 & 20.33 & 1314 & negative & 6560 & 13.67 \\
\hline 15 & 33.08 & 3283 & 13.00 & 1867 & 20.00 & 3869 & 12.67 \\
\hline 16 & 34.18 & 1096 & negative & 1372 & negative & 1881 & 12.00 \\
\hline 17 & 34.88 & 1082 & negative & 979 & negative & 1182 & negative \\
\hline 18 & 35.60 & 995 & negative & 1145 & negative & 916 & negative \\
\hline 19 & 35.87 & 1289 & negative & 834 & negative & 968 & negative \\
\hline 20 & 37.68 & 1290 & negative & 1457 & negative & 1683 & negative \\
\hline 21 & 37.84 & 1146 & negative & 1610 & negative & 2812 & negative \\
\hline
\end{tabular}

\section{Discussion And Conclusions}

A nucleic acid amplification enhancer is a reagent that can improve the efficiency, specificity, and yield of nucleic acid amplification. Several reports on PCR enhancers are available. For example, betaine has been observed to assist in the opening of double-stranded templates with high GC contents during denaturation 
by reducing the template sequence melting temperature $[12,13]$. Dimethyl sulfoxide (DMSO) reduces the need for interchain or intra-strand reannealing to improve the specificity and productivity of PCR reactions $[13,14]$. Glycerol $[14]$, trehalose [15], and formamide $[16,17]$ have also been reported to enhance PCR amplification.

Reports on amplification enhancers used in isothermal nucleic acid amplification are scarce. In the present work, we explored two nucleic acid amplification enhancers, namely, betaine and pullulan, and demonstrated their effectiveness as additives for RAA isothermal nucleic acid detection. Our results showed that, compared with the original RAA fluorescence method, the two enhancers (i.e., B-RAA and PRAA) could shorten the TT value, reduce the peak time, and decrease the detection time. Moreover, these enhancers could enhance the fluorescence value and increase the number of amplification products. Compared with that of the original RAA basic method, the sensitivity of B-RAA and P-RAA is 10-fold greater and their non-specific amplification is lower. Our results also indicated that betaine has better amplification enhancement effects than pullulan in the RAA assay for HPV18 (i.e., short fragment, 251 bp).

Betaine can help DNA polymerase pass through some complex secondary structures smoothly, thereby eliminating the pause in the extension process caused by the formation of secondary structures [12-14, 18]. Given the long length of RAA probes and primers, secondary structures within the primers, probes and DNA template may easily be formed by winding. We speculate that betaine relaxes these secondary structures, thereby facilitating RAA. Pullulan is a water-soluble microbial polysaccharide produced by Aureobasidium pullulans fermentation. Wang $\mathrm{R}$ et al. used pullulan as an accelerator in cross-priming amplification (CPA) and found that 1\% pullulan could increase the reaction rate of CPA by approximately 7 min. Some researchers have speculated that the addition of pullulan to the nucleic acid amplification system can improve the thermal stability of the polymerase and maintain its high activity, thus improving amplification efficiency [11]. The RAA system includes two types of enzymes, namely, recombinant enzyme UvsX and DNA polymerase, and the stability and activity of these enzymes are critical for amplification. The enhancement effect in our P-RAA study demonstrates that pullulan may exert a certain protective effect on the conformation of the enzyme, thereby enhancing the stability of the enzyme and the extent of amplification [11].

In the conventional (original) RAA method, the length of the amplicon is favorable when in the range of 100-200 bp; because amplicon lengths greater than $300 \mathrm{bp}$ are rarely reported, the application of RAA to amplicon enrichment for sequencing is seriously hindered. In the present study, we first observed that two enhancers could enhance the amplification efficiency of RAA. Thus, we applied these enhancers to the amplification of long fragments (509 bp). Our data showed that pullulan could improve the amplification efficiency of long fragments in the RAA fluorescence method. While betaine and pullulan can enhance the amplification of short fragments, the former does not play a significant role in the amplification of long fragments and may sometimes even inhibit RAA amplification. The specific reasons behind this finding require further investigation. We thus recommend pullulan as an additive to RAA assays for the amplification of long fragments. However, during conventional long-fragment amplification, the sensitivity 
of P-RAA is not much greater compared with that of the original RAA assay. This may be since the sensitivity of the RAA basic method is 10-100 times lower than that of the RAA fluorescence method, and the extension of such a long-fragment leads to such a result.

We tested various concentrations of trehalose, polyethylene glycol, DMSO, and bovine albumin as nucleic acid amplification enhancers for RAA assay in this study, and the improvements we observed were not as notable as those produced by betaine and pullulan (data not shown).

In summary, we found that two nucleic acid amplification enhancers, namely, betaine, and pullulan, are effective additives for RAA assays. If shorter fragments are to be amplified via the RAA method, $0.2,0.4$, or $0.6 \mathrm{M}$ betaine may be recommended; if longer fragments are to be amplified, $10 \%$ pullulan is recommended. However, we only performed the RAA assay for HPV 18 as an example and did not carry out experiments to detect other pathogens. The concentration and amount of enhancers may need to be adjusted within a specific range to avoid inhibitory effects. The optimal working concentrations of betaine or pullulan must be determined through further experiments when RAA assay is to be applied to detect other pathogens.

\section{Declarations}

\section{Funding}

This work was supported by the Youth Foundation of Academician Hou Yunde [grant number 2019HYDQNJJ03]; China Mega-Projects for Infectious Disease [grant number 2017ZX10302301

-004-002]

\section{Authors' contributions}

Jinrong Wang: Conceptualization, Data curation, Roles/Writing - original draft. Guowei Song: Formal analysis, Validation. Yue Ming: Conceptualization. Jing Pan, Ruiqing Zhang, Guohao Fan, and Yuan Gao: Software, Formal analysis. Xinxin Shen: Methodology, Supervision. Xuejun Ma: Funding acquisition, Project administration, Writing - review \& editing. Lixin Li: Conceptualization, Resources.

\section{Declaration of competing interest}

The authors declare no conflict of interest.

\section{References}

[1] C. Chen, X. Li, G. Li, L. Zhao, S. Duan, T. Yan, Z. Feng, X. Ma, Use of a rapid reverse-transcription recombinase aided amplification assay for respiratory syncytial virus detection, Diagnostic Microbiology and Infectious Disease, 90 (2018) 90-95. 
[2] G. Xue, S. Li, W. Zhang, B. Du, J. Cui, C. Yan, L. Huang, L. Chen, L. Zhao, Y. Sun, N. Li, H. Zhao, Y. Feng, Z. Wang, S. Liu, Q. Zhang, X. Xie, D. Liu, H. Yao, J. Yuan, Reverse-Transcription Recombinase-Aided Amplification Assay for Rapid Detection of the 2019 Novel Coronavirus (SARS-CoV-2), Analytical Chemistry, 92 (2020) 9699-9705.

[3] T. Wu, Y. Ge, K. Zhao, X. Zhu, Y. Chen, B. Wu, F. Zhu, B. Zhu, L. Cui, A reverse-transcription recombinaseaided amplification assay for the rapid detection of $\mathrm{N}$ gene of severe acute respiratory syndrome coronavirus 2 (SARS-CoV-2), Virology, 549 (2020) 1-4.

[4] J. Wang, K. Cai, X. He, X. Shen, J. Wang, J. Liu, J. Xu, F. Qiu, W. Lei, L. Cui, Y. Ge, T. Wu, Y. Zhang, H. Yan, Y. Chen, J. Yu, X. Ma, H. Shi, R. Zhang, X. Li, Y. Gao, P. Niu, W. Tan, G. Wu, Y. Jiang, W. Xu, X. Ma, Multiplecentre clinical evaluation of an ultrafast single-tube assay for SARS-CoV-2 RNA, Clin Microbiol Infect, 26 (2020) 1076-1081.

[5] R. Zhang, G. Li, X. Li, X. Shen, Y. Gao, L. Wang, T. Fan, Q. Duan, Y. Wang, J. Wang, Z.- Feng, X. Ma, A rapid and sensitive recombinase aided amplification assay incorporating competitive internal control to detect Bordetella pertussis using the DNA obtained by boiling, International Journal of Infectious Diseases, 86 (2019) 108-113.

[6] R. Wang, H. Zhang, Y. Zhang, X. Li, X. Shen, J. Qi, G. Fan, X. Xiang, Z. Zhan, Z. Chen, X. Ma, Development and evaluation of recombinase-aided amplification assays incorporating competitive internal controls for detection of human adenovirus serotypes 3 and 7, Virology Journal, 16 (2019).

[7] X. Bai, X. Ma, M. Li, X. Li, G. Fan, R. Zhang, R. Wang, Q. Duan, X. Shen, Y. Xie, X. Rong, Field applicable detection of hepatitis $B$ virus using internal controlled duplex recombinase-aided amplification assay and lateral flow dipstick assay, J Med Virol, (2020).

[8] X. Li, X. Shen, M. Li, J. Qi, R. Wang, Q. Duan, R. Zhang, T. Fan, X. Bai, G. Fan, Y. Xie, X. Ma, Applicability of duplex real time and lateral flow strip reverse-transcription recombinase aided amplification assays for the detection of Enterovirus 71 and Coxsackievirus A16, Virol J, 16 (2019) 166.

[9] G. Fan, X. Shen, F. Li, X. Li, X. Bai, R. Zhang, R. Wang, W. Lei, H. Wang, X. Ma, G. Wu, Development of an Internally Controlled Reverse Transcription Recombinase-aided Amplification Assay for the Rapid and Visual Detection of West Nile Virus, Biomed Environ Sci, 32 (2019) 926-929.

[10] J. Wang, J. Liu, G. Song, Z. Cao, J. Pan, X. Li, Y. Gao, J. Qi, Z. Chen, G. Fan, X. Bai, R. Zhang, R. Wang, Q. Duan, L. Li, X. Shen, X. Ma, Internally controlled recombinase-aided amplification (IC-RAA) assays for the detection of human papillomavirus genotypes 16 and 18 using extracted DNA and samples treated with nucleic acid releasing agent, Arch Virol, (2020).

[11] R. Wang, J. Wu, F. Zhang, L. Wang, F. Ji, On-point detection of GM rice in 20 minutes with pullulan as CPA acceleration additive, Anal. Methods, 6 (2014) 9198-9201. 
[12]D. Mytelka, M Chamberlin, Analysis and suppression of DNA polymerase pauses associated with a trinucleotide consensus, Nucleic Acids Res,24 (1996) 2774-2781.

[13] Michael A. Jensen, Marilyn Fukushima, R.W. Davis, DMSO and Betaine greatly improve amplification of GC-rich construvts in de novo synthesis., PLoS One, 5 (2010) e11024.

[14] V. Jurišić, J. Obradović, N. Tošić, S. Pavlović, M. Kulić, N. Djordjević, Effects of DMSO, glycerol, betaine and their combinations in detecting single nucleotide polymorphisms of epidermal growth factor receptor (EGFR) gene promoter sequence in non-small-cell lung cancer (NSCLC) patients, Journal of Pharmaceutical and Biomedical Analysis, 128 (2016) 275-279.

[15] N.M. Andrej-Niko lai Spiess, Richard Ivell, Trehalose is a potent PCR enhancer: lowering of DNA melting temperature and thermal stabilization of taq polymerase by the disaccharide trehalose, Clinical Chemistry, 50 (2004) 1253-1256.

[16] Gobinda Sarkar, Stephen Kapelner, S. S.Sommer, Formamide can dramatically improve the specificity of PCR., Nucleic Acids Research, 18 (1990) 7465.

[17] C. Raj, E.S. Clarence, The enhancement of PCR amplification by low molecular weight amides., Nucleic Acids Research, 29 (2001) 2377-12381.

[18] T. Yi, H. Zhang, H. Liang, G. Gong, Y. Cai, Betaine-assisted recombinase polymerase assay for rapid hepatitis B virus detection, Biotechnology and Applied Biochemistry, (2020).

\section{Figures}




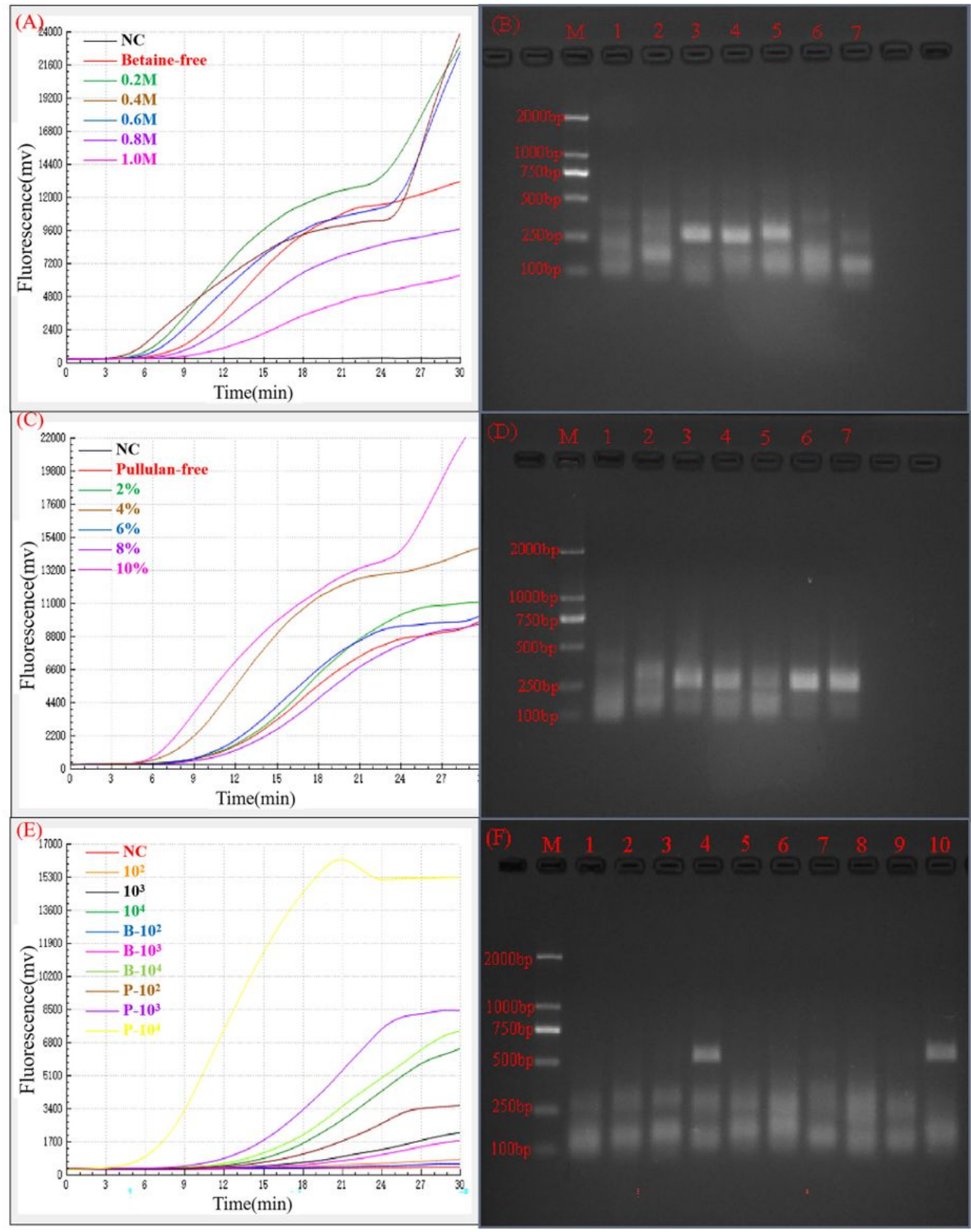

\section{Figure 1}

(A) B-RAA fluorescence amplification results of 10 copies/ $\mu \mathrm{L}$ HPV 18 plasmids with different concentrations of betaine. (B) B-RAA basic amplification results of 10 copies/ $\mu \mathrm{L}$ HPV 18 plasmids with different concentrations of betaine. Lane M: Marker, Lane 1-7: negative control, betaine-free, $0.2 \mathrm{M}$ betaine, $0.4 \mathrm{M}$ betaine, $0.6 \mathrm{M}$ betaine, $0.8 \mathrm{M}$ betaine, $1.0 \mathrm{M}$ betaine. (C) P-RAA fluorescence amplification results of 10 copies/ $\mu \mathrm{L}$ HPV 18 plasmids with different concentrations of pullulan. (D) P-RAA basic 
amplification results of 10 copies/ $\mu \mathrm{L}$ HPV 18 plasmids with different concentrations of pullulan. Lane M: Marker; Lane 1-7: negative control, pullulan-free, $2 \%$ pullulan, $4 \%$ pullulan, $6 \%$ pullulan, $8 \%$ pullulan, $10 \%$ pullulan. (E) RAA fluorescence long-fragment amplification results of 102-104 copies/ $\mu \mathrm{L}$ HPV18 plasmids with the original RAA, B-RAA, and P-RAA assays. (F) RAA basic long-fragment amplification results of 102-104 copies/ $\mu \mathrm{L}$ HPV18 plasmids with the original RAA, B-RAA, and P-RAA assays. Lane 1: negative control; Lanes 2-4: Amplification of 102-104 copies/ $\mu L$ HPV18 plasmids by the original RAA. Lanes 5-7: Amplification of 102-104 copies/ $\mu$ L HPV18 plasmids by B-RAA. Lanes 8-10: Amplification of 102-104 copies/ $\mu \mathrm{L}$ HPV18 plasmids by P-RAA. 